\title{
The use of mobile computing devices in microsurgery
}

\author{
Georgios Pafitanis ${ }^{1,2}$, Michalis Hadjiandreou ${ }^{3}$, Robert Miller ${ }^{1}$, Katrina Mason ${ }^{4}$, \\ Evgenia Theodorakopoulou ${ }^{5}$, Amir Sadri $^{2}$, Kirsten Taylor ${ }^{6}$, Simon Myers ${ }^{1}$ \\ ${ }^{1}$ Group for Academic Plastic Surgery, The Royal London Hospital, Barts Health NHS Trust, Queen Mary University of London, London; \\ ${ }^{2}$ Department of Plastic Surgery, Great Ormond Street Hospital for Children, London; ${ }^{3}$ Barts and The London School of Medicine and \\ Dentistry, London; ${ }^{4}$ Department of Ear, Nose and Throat Surgery, University Hospital Lewisham, London; ${ }^{5}$ Department of Plastic Surgery, \\ Norfolk and Norwich University Hospitals, Norwich; ${ }^{6}$ Department of Plastic Surgery, St. George's Hospital, London, UK
}

Mobile computing devices (MCDs), such as smartphones and tablets, are revolutionizing medical practice. These devices are almost universally available and offer a multitude of capabilities, including online features, streaming capabilities, high-quality cameras, and numerous applications. Within the surgical field, MCDs are increasingly being used for simulations. Microsurgery is an expanding field of surgery that presents unique challenges to both trainees and trainers. Simulation-based training and assessment in microsurgery currently play an integral role in the preparation of trainee surgeons in a safe and informative environment. MCDs address these challenges in a novel way by providing valuable adjuncts to microsurgical training, assessment, and clinical practice through low-cost, effective, and widely accessible solutions. Herein, we present a review of the capabilities, accessibility, and relevance of MCDs for technical skills acquisition, training, and clinical microsurgery practice, and consider the possibility of their wider use in the future of microsurgical training and education.

Keywords Microsurgery / Education, medical, continuing / Teaching

Received: 14 Feb 2018 - Revised: 17 Feb 2019 • Accepted: 27 Feb 2019

pISSN: 2234-6163 • elSSN: 2234-6171 • https://doi.org/10.5999/aps.2018.00150 • Arch Plast Surg 2019;46:102-107
Correspondence: Georgios Pafitanis Group for Academic Plastic Surgery, The Royal London Hospital, Barts Health NHS Trust, Queen Mary University of London, 4 Newark Street, Whitechapel, London E12AT, UK

Tel: +44-2078827173

E-mail: g.pafitanis@qmul.ac.uk

\section{INTRODUCTION}

Mobile computing devices (MCDs) combine powerful processors, operating systems, and communication features in the form of a single, hand-held, and pocket-stored device, allowing easy access and flexible use in healthcare environments. Smartphones and tablet computers have advanced features including web browsing, high-quality cameras, and access to numerous applications (apps), making them appealing for use in the healthcare setting. MCDs and apps are increasingly used by healthcare professionals for information management (writing and dictating notes), time management (scheduling appointments and meetings), health record maintenance and access (electronic prescribing), communications and consulting (video conferencing and social networking), reference and information gathering (medical journals, medical news, and drug reference guides), patient monitoring (collecting clinical data), clinical decision-making (clinical treatment guidelines), and medical education and training (board exam preparation, surgical simulations, and case study sharing).

Microsurgical training is relevant across several surgical specialties, including neurosurgery, ophthalmology, oral and maxil- 
lofacial surgery, ear nose and throat surgery, and-most notablyplastic surgery. It is widely accepted that microsurgical techniques are technically challenging to master. There are several reasons for this, including the difficulties in handling delicate tissues, the use of fine instruments (with a tip precision of 0.025 $\mathrm{mm}$ ), and complexities of the operating microscope. Microsurgical procedures require a high level of dexterity, relying particularly on the intrinsic muscles of the hand and a specific visuospatial awareness [1].

The learning curve in microsurgical skill acquisition is steep, and most Western services have seen a reduction in trainees' clinical exposure $[2,3]$, compounded by increasing pressures to ensure safe clinical training with objective measures. These factors have driven the development of simulation models. However, simulations can be expensive due to the costs of microscopic equipment and training facilities. Novel, low-cost, simulation training methods using MCDs, such as smartphones and tablet computers, are being increasingly explored to facilitate access to microsurgical training and education.

The aim of this paper is to present a comprehensive review of the use of MCDs in microsurgery. We review the capabilities, accessibility, and relevance of MCDs in technical skills acquisition, training, and clinical practice.

\section{METHODS}

Current useful applications of MCDs relevant to modern microsurgery practice were identified and analyzed in this comprehensive review. Each use or application was demonstrated, described and assigned a degree of relevance (low, moderate, and high) to three domains of modern microsurgery practice: technical skills acquisition, theoretical education, and clinical practice (Table 1). Each assigned use or application was further ana- lyzed according to the relevant domains of modern microsurgery practice in a simulated training environment and in clinical practice, with the goal of developing an easy-to-access guide for future innovations.

\section{RESULTS}

Eleven uses and applications of MCDs were identified, of which nine had some degree of relevance to all three domains of modern microsurgery practice. Only two uses demonstrated some degree of relevance to fewer than three domains of modern microsurgery practice. The domain of clinical microsurgery practice had five high-relevance uses/applications, the domain of theoretical education had four high-relevance uses/applications, and the domain of technical skills acquisition had three high-relevance uses/applications. Only one use (recording of microsurgical techniques using a high-definition camera) demonstrated high relevance in all three domains of modern microsurgery practice. Table 1 presents a further analysis and evaluation of the value of those uses/applications across the three domains of modern microsurgery practice.

\section{DISCUSSION}

\section{MCDs in microsurgical skill training}

MCDs have several uses within microsurgical simulation and training. These span from theoretical knowledge to discussion platforms and low-cost training platforms.

\section{Theoretical microsurgery knowledge acquisition and discussion} Smartphones can help the microsurgery trainee acquire theoretical knowledge before beginning to train using microscopy. Detailed information about microsurgery instruments, sutures,

\section{Table 1. Classification of the use and relevant applications of MCDs in three relevant categories}

\section{Use of MCDs in microsurgery}

\section{Technical skills acquisition}

Theoretical microsurgery knowledge acquisition through SMaRT app Clinical simulation apps such as TouchSurgery \& FlapApp

Sharing of skill training tips on social media platforms

Recording of microsurgical techniques using high-definition camera

Projecting a microsurgical procedure on a larger display

Live-streaming microsurgical procedure to trainees

Microsurgical simulation using a cheap, foldable simulator such as eoMicro

Using MCD as end-product assessment tool

Free video analysis app (Ubersense) to analyze microsurgical skills

Free flap postoperative perfusion monitoring by colorimetry (SlipaRamanitor)

Free flap postoperative perfusion monitoring by thermography (FLIR ONE)

\begin{tabular}{ccc|}
+ & +++ & practice \\
+ & ++ & ++ \\
+ & ++ & + \\
++ & +++ & +++ \\
+ & +++ & +++ \\
+ & +++ & +++ \\
+++ & ++ & + \\
+++ & ++ & + \\
+++ & + & ++ \\
- & + & +++ \\
- & ++ & +++
\end{tabular}

The symbols in the table indicate low relevance (+), moderate relevance (++), and high relevance (+++).

MCD, mobile computing device. 
the microscope setup, and body positioning can be found in the Stanford Microsurgery and Resident Training (SMaRT) app, which can be downloaded gratis on a smartphone [4]. Trainees can find multiple videos of basic microsurgery training, such as Acland's microsurgery video series, on YouTube and use them to familiarize themselves with relevant techniques before practicing under the microscope. Furthermore, Google Play and the App Store have numerous medical apps that break down microsurgical procedures into smaller steps to aid learning. Touch Surgery is an interactive surgical simulator that enables the user to undertake microsurgical operations in realistic 3-dimensional surgical fields. FlapApp focuses on educating the user regarding appropriate patient selection for microsurgical procedures, such as microsurgical breast reconstruction, and highlights the operative steps with clinical images and drawings [5]. In addition to the above apps, MCDs are excellent tools to access social media and communicate with experts and trainees in the field of microsurgery. The International Microsurgery Club (IMC) is a comprehensive social media-based platform that facilitates sharing of technical experience and advice among international microsurgeons, discussion of interesting cases, online paper-writing collaborations, and promotion of microsurgery meetings and events. The IMC serves as an excellent resource for any trainee for complex case discussions with global expert input and interaction [6].

Furthermore, telemedicine systems allow users to utilize video chat service applications for clinical off-site consultations or provide platforms for tele-education. MCDs also function as a handy tool for providing telemedicine solutions in rural areas and war zones, such as for limb salvage procedures when local expertise is not available. This tool offers the advantages of ease of use, high portability, and low cost [7]. It is important to note that online medical information sharing should always happen under the condition that any user must abide by the data and information sharing regulations of their employer.

\section{Training platforms and simulation}

MCDs offer the ability to project microsurgical operations on a larger display using wired technology, such as an HDMI cable, or high-speed Wi-Fi technology. This holds significant educational value, especially with a group of trainees. A microscope adapter that universally adjusts to any smartphone is commercially available and can be used to keep the smartphone steady in position. Multiple adapters can be used to project multiple operations simultaneously. The approach enables real-time viewing of operative techniques, observation of on-table skills and challenges, and case-based teaching. Fig. 1 demonstrates this proposed use [8]. Furthermore, MCDs offer advantages in

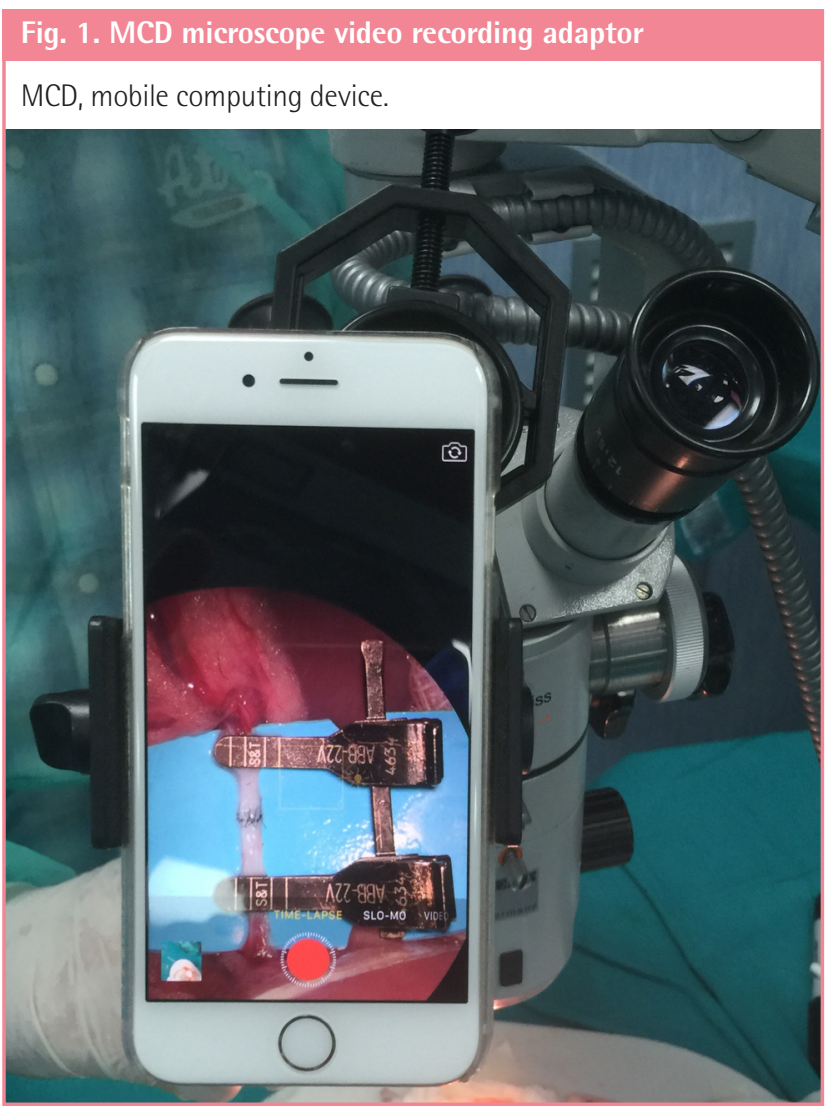

training surgeons more efficiently and in larger groups and, as mentioned above, telemedicine can be used to bridge inequalities in education and training in remote areas. In addition to projecting footage onto larger displays, smartphones can broadcast live microsurgical procedures to many trainees (with limitations for animal simulations, and only with informed consent and confidentiality control for clinical broadcasts). Legal and ethical concerns around privacy, information sharing, publicity, and intellectual property of recorded material should be addressed within the host organization's protocols for digital use and sharing.

Furthermore, MCDs can bypass the inherent limitations of cost and access of traditional microsurgical lab-based training. The accessibility allows training in any location that suits the trainee. A 2015 study by Kim et al. [9] demonstrated that smartphones could enlarge the operative field to a maximum of 3-fold (iPhone 5S, Apple Inc.) or 4-fold (Galaxy S4, Samsung), a size that trainees found adequate for practicing microsurgical endto-end anastomoses using 8-0 suture material. Trainees were confident and eager to continue practicing under the smartphone camera magnification system. Furthermore, simulators have been developed to allow microsurgical skill acquisition using a cost-effective and affordable platform with a smartphone: the eoMicro Simulator (Fig. 2). Low-fidelity models have been 


\section{Fig. 2. eoMicro simulator}

The eoMicro simulator in combination with a smartphone creates a home microsurgical set-up that allows microsurgical skills improvement [10].

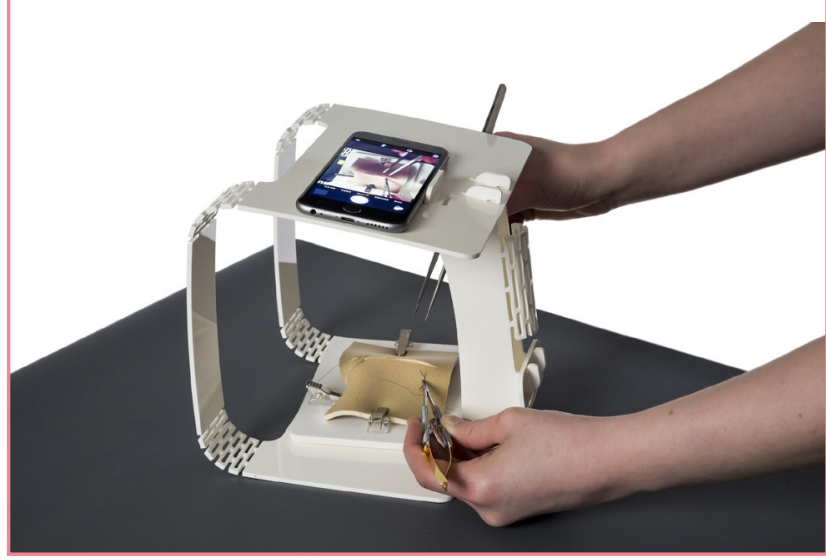

established as an effective method of surgical skill acquisition during the early phase of the learning curve, as their use in selfdirected deliberate practice has been shown to improve technical performance. The eoMicro simulator is a home microsurgical set-up, utilizing a portable flat-pack Perspex designed platform that allows the trainee to use it in combination with a smartphone to perform surgical tasks and improve his or her microsurgical skills [10].

\section{MCDs in microsurgical skill assessment}

The use of MCDs in microsurgery has been widely available; however, no comprehensive review has been performed of the current available applications and practices to evaluate and demonstrate those capabilities. High-definition cameras are widely available on mobile devices. A simple and cost-effective method of video-recording can facilitate the objective assessment of microsurgical technical skills, using instruments such as validated global rating scales (GRS). A mobile device can be coupled to the operating microscope using a commercially available adaptor, allowing the recording of high-quality videos [8]. Videos can then be scored by experts using a modified GRS (videomodified Objective Structured Assessment of Technical Skills; OSATS) for economy of movement, confidence of movement, respect for tissue, and performance of specific technical steps. Furthermore, end-product assessment tools are increasingly used to objectively evaluate the quality of microvascular anastomoses, and to follow progression along the learning curve in early microsurgery training. The anastomosis lapse index (ALI) is used to evaluate microvascular anastomosis skill in living and non-living simulation models, with predictive validity in the latter. Disruptions of the anastomotic line, oblique stitches, partial thickness stitches, and vessel tears are identified in the intimal surface of a completed anastomosis that has been excised, divided longitudinally, and laid open flat. A smartphone can take a high-quality image of the prepared anastomosis down the microscope eyepiece, and this image can be forwarded electronically to the assessor. The frequency and type of error define a skill level in the validated ALI tool [11].

Ubersense (Hudl Technique: Slow Motion Video Analysis, Ubersense Inc.) is a smartphone application, initially designed for athletes and coaches, that allows slow-motion analysis of movement frame-by-frame. A video can be trimmed, compared side-by-side with another video by drawing and parameter analysis, and shared with other users. The needle-to-needle driver angle, needle tip-to-needle driver distance, distance of suture entry and exit in relationship to the vessel edge, needle-to-vessel wall angle, suture tail length, number of ancillary movements, and time to completion can all be measured and analyzed to show skill acquisition. A trainer can identify areas of weakness and potential opportunities for improvement. The technology is easily accessed and comes at no cost for the individual trainee or the institution or laboratory [12]. MCDs also offer opportunities for trainee self-assessment and evaluation. As surgical training programs embrace objective skill assessment, standardization is required. However, valid and low-cost tools for selfevaluation/improvement are not yet commonplace.

\section{MCDs in microsurgical clinical practice}

MCDs offer many advantages for microsurgical training and assessment. Moreover, important advances are also occurring in the use of MCDs for the clinical management of patients undergoing microsurgical procedures.

\section{The use of MCDs in postoperative flap monitoring}

Free flaps have become the reconstruction of choice for many soft tissue defects. Arterial occlusion and/or venous insufficiency can lead to partial or total flap failure over the first 5 postoperative days, and close observation over this period is important for flap salvage [13]. Many devices are complicated to use and expensive, and therefore are not routinely used in the United Kingdom, where labor-intensive clinical observation remains the mainstay. The Android diagnostic application SlipaRamanitor recognizes skin color characteristics from skin images acquired using a smartphone and a custom-made light box, with high diagnostic accuracy (95\%) [14]. It is reliable and inexpensive for both the clinical and non-clinical learning environment [14]. Whilst there is no substitute for clinical diagnosis, MCDs can contribute to flap monitoring, especially when reliant on relatively inexperienced first responders. They can also be used in this way as an end-product assessment and feedback tool in 


\section{Fig. 3. FLIR ONE thermal imaging MCD adaptor}

FLIR ONE: a smartphone-compatible miniature thermal imaging camera that digitally merges a light camera photograph with a thermal image [15]. MCD, mobile computing device.

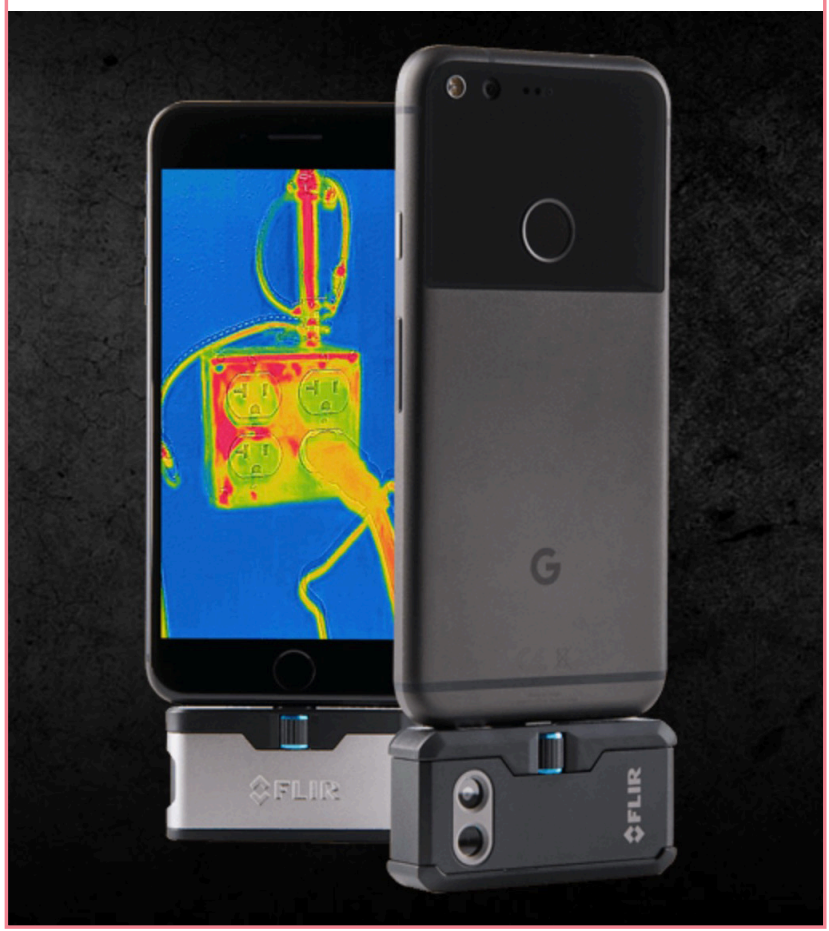

non-clinical in vivo simulations. In addition to the SlipaRamanitor application discussed above, there are monitoring tools that allow postoperative flap tissue perfusion analysis through thermography. In these tools, infrared radiation from an object is detected to generate an image based on the local temperature, which can also be used as an effective marker of cutaneous blood flow. Traditionally, high-resolution thermal cameras are expensive. FLIR ONE (Fig. 3) is a smartphone-compatible miniature thermal imaging camera that is compact, less expensive than traditional cameras, and requires minimal training. A visible light camera photograph is digitally merged with a thermal image [15]. However, currently, the FLIR ONE static thermal imaging is not as reliable for perforator mapping (for in vivo perforator flap planning) as dynamic infrared thermography.

\section{NOTES}

\section{Conflict of interest}

No potential conflict of interest relevant to this article was reported.

\section{Author contribution}

Study concept and design: Pafitanis G. Data acquisition: Pafitanis G, Myers S. Data analysis and interpretation: Pafitanis G,
Hadjiandreou M, Miller R, Myers S. Drafting of the manuscript: Pafitanis G, Hadjiandreou M, Miller R, Taylor K. Critical revision of the manuscript for important intellectual content: all authors. Administrative: Pafitanis G, Hadjiandreou M. Technical or material support: Hadjiandreou M, Sadri A. Approval of the final manuscript: all authors.

\section{ORCID}

Georgios Pafitanis https://orcid.org/0000-0003-4712-277X

Michalis Hadjiandreou https://orcid.org/0000-0002-25357896

Katrina Mason https://orcid.org/0000-0002-4515-1780

Evgenia Theodorakopoulou https://orcid.org/0000-00028283-0483

Simon Myers https://orcid.org/0000-0002-4435-0390

\section{REFERENCES}

1. Chan W, Niranjan N, Ramakrishnan V. Structured assessment of microsurgery skills in the clinical setting. J Plast Reconstr Aesthet Surg 2010;63:1329-34.

2. Sadideen H, Kneebone R. Practical skills teaching in contemporary surgical education: how can educational theory be applied to promote effective learning? Am J Surg 2012; 204:396-401.

3. Ghanem AM, Hachach-Haram N, Leung CC, et al. A systematic review of evidence for education and training interventions in microsurgery. Arch Plast Surg 2013;40:312-9.

4. Vargo J. Stanford Microsurgery and Resident Training [Internet]. Top Plastic Surgery Apps, c2017 [cited 2019 Mar 12]. Available from: http://topplasticsurgeryapps.com/2016/07/ 25/stanford-microsurgery-and-resident-training/.

5. Mosa AS, Yoo I, Sheets L. A systematic review of healthcare applications for smartphones. BMC Med Inform Decis Mak 2012;12:67.

6. SciTeMed Publishing Group. A new platform for global microsurgeons [Internet]. Cheyenne, WY: SciTeMed Publishing Group; c2017 [cited 2019 Mar 12]. Available from: https://www.scitemed.com/Press/10/A-new-platform-forglobal-microsurgeons.

7. Hsieh CH, Tsai HH, Yin JW, et al. Teleconsultation with the mobile camera-phone in digital soft-tissue injury: a feasibility study. Plast Reconstr Surg 2004;114:1776-82.

8. Perry D, Albert M, Akyurek M. Use of smartphone cameras for simplified and cost-effective video recording of microvascular techniques. Plast Reconstr Surg 2015;135:941e943e.

9. Kim DM, Kang JW, Kim JK, et al. Microsurgery training us- 
ing a smartphone. Microsurgery 2015;35:500-1.

10. eoSurgical. eoMicro [Internet]. Edinburgh, eoSurgical [cited 2019 Mar 12]. Available from: https://www.eosurgical. com/products/eomicro.

11. Ghanem AM, Al Omran Y, Shatta B, et al. Anastomosis lapse index (ALI): a validated end product assessment tool for simulation microsurgery training. J Reconstr Microsurg 2016;32:233-41.

12. Shah A, Rowlands M, Patel A, et al. Ubersense: using a free video analysis app to evaluate and improve microsurgical skills. Plast Reconstr Surg 2014;134:338e-339e.

13. Smit JM, Acosta R, Zeebregts CJ, et al. Early reintervention of compromised free flaps improves success rate. Microsurgery 2007;27:612-6.

14. Kiranantawat K, Sitpahul N, Taeprasartsit P, et al. The first smartphone application for microsurgery monitoring: SilpaRamanitor. Plast Reconstr Surg 2014;134:130-9.

15. Hardwicke JT, Osmani O, Skillman JM. Detection of perforators using smartphone thermal imaging. Plast Reconstr Surg 2016;137:39-41. 